\title{
NONLINEAR FILTERING OF A SYSTEM OF LOGISTIC EQUATIONS
}

\author{
REHEZ AhLiP AND Vo ANH
}

\begin{abstract}
This paper is concerned with the filtering problem for a nonlinear stochastic system of prey-predator logistic equations. Based on the innovations approach, we establish the Zakai equation for the unnormalised conditional distribution and the adjoint Zakai equation for the unnormalised conditional density of the nonlinear filter. Using a perturbation technique, we obtain the appropriate expressions for the unnormalised conditional distribution and density of stochastic integrals with respect to the observation processes.
\end{abstract}

\section{INTRODUCTION}

In studies of population dynamics, the state of a system is commonly described by a logistic-type equation:

$$
d N_{t}=A N_{t}\left(1-B N_{t}\right) d t .
$$

Here, $N_{t}$ denotes the population density and $A$ the initial per capita growth rate, while $B$ is the reciprocal of the environmental carrying capacity. A more realistic model to describe the state of the system is to include a stochastic element in (1.1) so that the system under investigation is given by the following stochastic differential equation:

$$
d N_{t}=A N_{t}\left(1-B N_{t}\right) d t+\sigma d W_{1}(t),
$$

where $W_{1}(t)$ is a Brownian motion.

It is a common situation in practice that the signal process $\left\{N_{t}\right\}$ cannot be observed directly, but must be estimated from observation on a related process $y_{t}$ which can be assumed to have the form

$$
d y_{t}=k N_{t} d t+\sigma_{l} d W_{2}(t),
$$

where $W_{2}(t)$ is another Brownian motion independent of $W_{1}(t)$. The objective then is to compute a least squares estimate of $N_{t}$ given the observations $\left\{y_{s}, 0 \leqslant s \leqslant t\right\}$. In

Received 2nd April, 1996

The authors wish to thank Professor RJ Elliott of the University of Alberta, Alberta, Canada, for his generously shared insights and assistance which led to the preparation of this paper.

Copyright Clearance Centre, Inc. Serial-fee code: 0004-9729/97 \$A2.00+0.00. 
addition, it is required that this computation be done recursively. This is the classical filtering problem.

In this paper, we consider the filtering of a nonlinear system of prey-predator competition in a stochastic environment given by:

$$
\begin{aligned}
& d N_{t}^{(1)}=N_{t}^{(1)}\left(A-B N_{t}^{(1)}-C N_{t}^{(2)}\right) d t+\varepsilon_{1} d W_{t}^{1}, \\
& d N_{t}^{(2)}=N_{1}^{2}\left(-D+E N_{t}^{(1)}\right) d t+\varepsilon_{2} d W_{t}^{2},
\end{aligned}
$$

where $N_{t}^{1}=$ prey population, $N_{1}^{2}=$ predator population, and the observation processes are given by:

$$
\begin{aligned}
& d y_{t}^{(1)}=k_{1} N_{t}^{(1)} d t+d B_{t}^{(1)}, \\
& d y_{t}^{(2)}=k_{2} N_{t}^{(2)} d t+d B_{t}^{(2)},
\end{aligned}
$$

where $W_{t}^{1}, W_{t}^{2}, B_{t}^{1}$ and $B_{t}^{2}$ are independent Brownian motions on a probability space $(\Omega, \mathfrak{F}, P)$ with a filtration $\left\{\mathfrak{F}_{t}\right\}_{t \geqslant 0}$.

The filtering problem for the logistic equation of a single species (system (1.1) and (1.2)) is solved in [4]. In this paper, we extend the results of [4] to the prey-predator system (1.3) and (1.4). As described in [3] and [4], nonlinear filtering can be approached via the innovations technique. We shall collect some key results of this technique in Section 2. Using the innovations technique, we obtain in Section 3 the Kushner equation for the conditional joint distribution of $N_{t}^{(1)}, N_{t}^{(2)}$ given the past history of $y_{t}$. The Kushner equation still poses two difficulties: It contains nonlinear terms and is driven by the innovations process. To overcome these difficulties, we obtain in Section 4 the Zakai equation for the unnormalised conditional distribution. The Zakai equation for our model consists of two stochastic integrals with respect to the observation processes. Using a pertubation technique of Kunita [6], we establish in Section 5 an expression for the unnormalised conditional distribution involving no stochastic integrals. Finally, in Section 6, we derive the adjoint Zakai equation for the unnormalised conditional density, which again can be transformed into an expression free of stochastic integrals by appealing to Kunita's technique.

The Zakai equation for the unnormalised conditional distribution extends the result of Elliott [4] in the single species case; while the multidimensional adjoint Zakai equation for the unnormalised conditional density of the system (1.3) and (1.4) is new. These results solve the nonlinear filtering problem for the system (1.3) and (1.4) of preypredator logistic equations completely.

\section{THE INNOVATIONS METHOD TO NONLINEAR FILTERING}

The basic references for this section are Davis and Marcus [2], Elliott [3, 4] and Kunita [6]. All stochastic processes will be defined on a fixed probability space $(\Omega, \mathfrak{F}, P)$ 
and a finite time interval $[0, T]$, on which there is defined an increasing family of $\sigma$-fields $\left\{\mathfrak{F}_{t}, 0 \leqslant t \leqslant T\right\}$. All processes considered will be $\mathfrak{F}_{t}$-adapted.

Consider a real-valued Markov process $\xi_{t}$ and the related observation process $y_{t}$ given by

$$
d y_{t}=h\left(\xi_{t}\right) d t+d B_{t},
$$

where $B_{t}$ is Brownian motion. We shall assume that $\xi_{t}$ is independent of the increments of $B_{t}$. Define $\mathfrak{Y}_{t}=\sigma\left\{y_{s}, 0 \leqslant s \leqslant t\right\}$ as the $\sigma$-field generated by the given observation process $y_{t}$. The objective is to compute in a recursive form an expression for the best estimate of $\xi_{t}$ given the history of the observations to time $t, \mathfrak{Y}_{t}$. That is, we want to obtain an expression for

$$
\widehat{\xi}_{t}=E\left[\xi_{t} \mid \mathfrak{Y}_{t}\right]
$$

Now, let us introduce the innovations process

$$
\nu_{t}=y_{t}-\int_{0}^{t} \widehat{h}\left(\xi_{s}\right) d s
$$

The incremental innovations $\nu_{t+h}-\nu_{t}$ represents the "new information" concerning the process $\left\{Z_{t}=h\left(\xi_{t}\right)\right\}$ available from the observations between $t$ and $t+h$, in the sense that $\nu_{t+h}-\nu_{t}$ is independent of $\mathfrak{Y}_{t}$.

The process $\nu_{t}$ has the following properties:

(i) The process $\left(\nu_{t}, \mathfrak{Y}_{t}\right)$ is a standard Brownian motion, that is $\nu_{t}$ is a $\mathfrak{Y}_{t^{-}}$ martingale and $\langle\nu\rangle_{t}=t$, where $\langle\nu\rangle$ is the unique predictable process such that $\nu^{2}-\langle\nu\rangle$ is a martingale.

(ii) Every square integrable martingale $\left(m_{t}, \mathfrak{Y}_{t}\right)$ with respect to the observation $\sigma$-field $\mathfrak{Y}_{t}$ is sample continuous and has the representation

$$
m_{t}=\int_{0}^{t} g_{s} d \nu_{\iota}
$$

where the process $g$ is $\mathfrak{Y}_{t}$-predictable. In other words, $\boldsymbol{m}_{\boldsymbol{t}}$ can be written as a stochastic integral with respect to the innovations process.

Suppose the signal process $\xi_{t}$ is a real-valued $\mathfrak{F}_{t}$-semimartingale of the form

$$
\xi_{t}=\xi_{0}+\int_{0}^{t} \alpha, d s+\eta_{t}
$$

where $\alpha$ is an $\mathfrak{F}$-adapted process such that

$$
E \int_{0}^{T} \alpha_{s}^{2} d s<\infty
$$


$\xi_{0}$ is an $\mathfrak{F}_{0}$-measurable random variable with $E \xi_{0}^{2}<\infty$, and $\eta_{t}$ is a square integrable $\mathfrak{F}_{t}$-martingale. There is a unique predictable process $\langle\eta, B\rangle$ such that $\left(\eta_{t} B_{t}-\langle\eta, B\rangle_{t}, \mathfrak{F}_{t}\right)$ is a martingale. Let us assume that this process is of the form $\langle\eta, B\rangle_{t}=\int_{0}^{t} \beta, d s$.

THEOREM 2.1. Write $\widehat{\xi}=E\left[\xi_{t} \mid \mathfrak{Y}_{t}\right]$ for the filtered estimate of $\xi_{t}$, given $\mathfrak{Y}_{t}$. Then $\left\{\widehat{\xi}_{t}\right\}$ satisfies the stochastic differential equation

$$
\widehat{\xi}_{t}=\widehat{\xi}_{0}+\int_{0}^{t} \widehat{\alpha}_{s} d s+\int_{0}^{t}\left(\widehat{\xi_{s} h}\left(x_{s}\right)-\widehat{\xi_{s}} \widehat{h}\left(x_{s}\right)+\widehat{\beta}_{s}\right) d \nu_{s}
$$

Proof: See Elliott [4].

Formula (2.6) is not a recursive equation for $\hat{\xi}_{t}$ and hence is not very useful as it stands, but can be used to obtain an infinite-dimensional recursive equation for the filtering problem.

COROLLARY 2.2. Let $\left\{x_{t}\right\}$ be the solution of the stochastic differential equation

$$
d x_{t}=f\left(t, x_{t}\right) d t+\sigma\left(x_{t}\right) d W_{t}
$$

that is,

$$
x_{t}=x_{0}+\int_{0}^{t} f\left(s, x_{s}\right) d s+\int_{0}^{t} \sigma\left(x_{s}\right) d W_{s},
$$

and let $Y_{t}$ be given by (2.1) with $\left\{W_{t}\right\}$ and $\left\{B_{t}\right\}$ being independent. Then for any function $F \in C^{2}, \Pi_{t}(F)=E\left[f\left(x_{t}\right) \mid \mathfrak{Y}_{t}\right]$ satisfies

$$
\Pi_{t}(F)=\int_{0}^{t} \Pi_{s}(L F) d s+\int_{0}^{t}\left[\Pi_{s}(F h)-\Pi_{s}(F) \Pi_{s}(h)\right] d \nu_{s},
$$

where

$$
L F(x)=f(s, x) F_{x}(x)+\frac{1}{2} \sigma(x)^{2} F_{x x}(x) .
$$

Proof: The Ito differential rule yields

$$
F\left(x_{t}\right)=F\left(x_{0}\right)+\int_{0}^{t} F_{x}\left(x_{s}\right) f\left(s, x_{s}\right) d s+\int_{0}^{t} F_{x}\left(x_{s}\right) \sigma\left(x_{s}\right) d W_{s}+\frac{1}{2} \int_{0}^{t} F_{x x}\left(x_{s}\right) \sigma\left(x_{s}\right)^{2} d s .
$$

Now applying Theorem 2.1 to the semimartingale $f\left(x_{t}\right)$ gives

$$
\Pi_{t}(F)=\Pi_{0}(F)+\int_{0}^{t} \Pi_{s}(L F) d s+\int_{0}^{t}\left[\Pi_{s}(F h)-\Pi_{s}(F) \Pi_{s}(h)\right] d \nu_{s} .
$$

Since $\left\{\Pi_{t}(F): F \in C^{2}\right\}$ determines a measure-valued stochastic process $\Pi_{t},(2.6)$ can be regarded as a recursive (infinite-dimensional) stochastic differential equation for the conditional measure $\Pi_{t}$ of $\xi_{t}$ given $\mathfrak{Y}_{t}$, and $\Pi_{t}(F)$ is a conditional statistic computed from $\Pi_{t}$ in a memoryless fashion. 
REMARK. Elliott [4] solved the filtering problem for the single species system (1.1) and (1.2) using these methods together with the perturbation technique of Kunita [6]. Whereas these sophisticated methods are appropriate for an $n$-dimensional system, $n \geqslant 2$, as can be seen in the next sections, the Kalman-Bucy filter for linear systems is adequate for the one-dimensional problem, if the observation process is formulated in an appropriate way. In fact, let us consider the following observation process:

$$
d Y_{t}=k\left(\frac{1}{B N_{t}}-1\right) d t+\sigma_{2} d W_{2}(t)
$$

(see Antonelli [1]). Using the substitution

$$
N^{*}=\frac{1}{B N_{t}}-1
$$

we obtain the following linear estimation problem:

$$
\begin{aligned}
d N_{t}^{*} & =-A N_{t}^{*} d t+\sigma_{1} d W_{1}(t), \\
d Y_{t} & =-k N_{t}^{*} d t+\sigma_{2} d W_{2}(t) .
\end{aligned}
$$

Now, to (2.13) and (2.14) we apply the Kalman-Bucy filtering method (see Lipster and Shiryayev [8]; Oksendal [9]) to obtain $\widehat{N}_{t}^{*}$, the conditional expectation of $N_{t}^{*}$ given the observations up to time $t$ on $\mathfrak{Y}_{t}$, and $S_{t}$ the mean square error estimate. Suppose that the initial distribution $\mathfrak{N}_{0}$ is Gaussian with zero mean and variance $S_{0}$. The Kalman-Bucy [5] equations are

$$
d \widehat{N}_{t}^{*}=\left(-A-\frac{k^{2} S(t)}{\sigma_{2}^{2}}\right) \widehat{N}_{t}^{*} d t+\frac{k S(t)}{\sigma_{2}^{2}} d y_{t},
$$

where $S(t)=E\left[\left(N_{t}^{*}-\widehat{N}_{t}^{*}\right)^{2} \mid \mathfrak{Y}_{t}\right]$ satisfies the (deterministic) Riccati equation

$$
\frac{d S}{d t}=-2 A S(t) \frac{k^{2}}{\sigma_{2}^{2}} S^{2}(t)+\sigma_{1}^{2}, S(0)=\alpha^{2},
$$

which has the solution

$$
S(t)=\frac{A_{1}-M \cdot A_{2} \exp \left(\frac{\left(A_{2}-A_{1}\right) k^{2} t}{\sigma_{2}^{2}}\right)}{1-M \cdot \exp \frac{\left(A_{2}-A_{1}\right) k^{2} t}{\sigma_{2}^{2}}},
$$


where

and

$$
\begin{aligned}
& A_{1}=\frac{1}{k_{2}}\left(-A \sigma_{2}^{2}-\sigma_{2} \sqrt{A^{2} \sigma_{2}^{2}+k^{2} \sigma_{1}^{2}}\right) \\
& A_{2}=k^{2}\left(-A \sigma_{2}^{2}-\sigma_{2} \sqrt{A^{2} \sigma_{2}^{2}+k^{2} \sigma_{1}^{2}}\right) \\
& M=\frac{\alpha^{2}-A_{1}}{\alpha^{2}-A_{2}}
\end{aligned}
$$

Hence we obtain the solution for $\widehat{N}_{t}^{*}$ in the form

$$
\widehat{N}_{t}^{*} \exp \left(\int_{0}^{t} G(s) d s\right) \hat{N}_{0}^{*}+\frac{k^{2}}{\sigma_{2}^{2}} \int_{0}^{t} \exp \left(\int_{0}^{t} G(u) d u\right) S(s) d y_{s}
$$

where

$$
G(s)=-A-\frac{k^{2}}{\sigma_{2}^{2}} S(s)
$$

3. NONLINEAR FILTERING OF A PREY-PREDATOR SYSTEM

Consider the system (1.3) and(1.4). Let $\Pi_{t}(N)=E\left[N_{t} \mid \mathfrak{Y}_{t}\right]$ where $\mathfrak{Y}_{t}=$ $\sigma\left\{y^{(1)}, y_{s}^{(2)}, x \leqslant t\right\}$. The innovations processes are

$$
\begin{aligned}
& \nu_{t}^{1}=y_{t}^{1}-\int_{0}^{t} k_{1} \Pi \cdot\left(N^{(1)}\right) d s, \\
& \nu_{t}^{2}=y_{t}^{2}-\int_{0}^{t} k_{2} \Pi \cdot\left(N^{(2)}\right) d s .
\end{aligned}
$$

Let $F: R^{2} \rightarrow R^{1}$ be any function in $C^{2}$. Using Ito's differential rule, we have

$$
\begin{aligned}
& F\left(N_{t}^{(1)}, N_{t}^{(2)}\right)=F\left(N_{0}^{(1)} N_{0}^{(2)}\right) \\
& \quad+\int_{0}^{t} \frac{\partial F}{\partial N^{(1)}} d N_{s}^{(1)}+\int_{0}^{t} \frac{\partial F}{\partial N^{(2)}} d N_{s}^{(2)}+\frac{1}{2} \int_{s}^{t} \varepsilon_{1}^{2} \frac{\partial^{2} F}{\partial N^{(1)^{2}}} d s+\frac{1}{2} \int_{0}^{t} \varepsilon_{2}^{2} \frac{\partial^{2} F}{\partial N^{(2)^{2}}} d s
\end{aligned}
$$

that is,

$$
\begin{aligned}
& F\left(N_{t}^{(1)}, N_{t}^{(2)}\right)=F\left(N^{(1)}, N_{0}^{(2)}\right) \\
& \quad+\int_{0}^{t} \frac{\partial F}{\partial N^{(1)}}\left(\left(A-B N_{a}^{(1)}-C N_{t}^{(2)}\right) N_{a}^{(1)}\right) d s+\int_{0}^{t} \frac{\partial F}{\partial N^{(2)}}\left(\left(-D+E N_{a}^{(1)}\right) N_{a}^{(2)}\right) d s \\
& \quad+\int_{0}^{t} \varepsilon_{1} \frac{\partial F}{\partial N_{t}^{(1)}} d W_{t}^{1}+\int_{0}^{t} \varepsilon_{2} \frac{\partial F}{\partial N_{a}^{(2)}} d W_{t}^{2}+\frac{1}{2} \int_{0}^{t}\left(\varepsilon_{1}^{2} \frac{\partial^{2} F}{\partial N^{(1) 2}}+\varepsilon_{2}^{2} \frac{\partial^{2} F}{\partial N^{(2) 2}}\right) d s .
\end{aligned}
$$


Let us use the notation

$$
\Pi_{t}(F)=E\left[F\left(N_{t}^{(1)}, N_{t}^{(2)}\right) \mid \mathfrak{Y}_{t}\right]
$$

Then $\Pi_{t}$ can be thought of as the conditional joint distribution of $N_{t}^{(1)}, N_{t}^{(2)}$ given $\mathfrak{Y}_{t}$. Since $W_{t}^{1}, W_{t}^{2}, B_{t}^{1}, B_{t}^{2}$ are independent, applying Theorem 2.1 to the semimartingale $F\left(N_{t}^{(1)}, N_{t}^{(2)}\right)$ gives

$$
\begin{aligned}
\Pi_{t}\left(F\left(N_{t}^{(1)}, N_{t}^{(2)}\right)\right)= & \Pi_{0}\left(F\left(N_{0}^{(1)}, N_{0}^{(2)}\right)\right) \\
& +\int_{0}^{t} \Pi_{s}\left(\frac{\partial F}{\partial N^{(1)}}\left(A-B N_{s}^{(1)}-C N_{s}^{(2)}\right) N_{s}^{(1)}\right) d s \\
& +\int_{0}^{t} \Pi_{s}\left(\frac{\partial F}{\partial N^{(2)}}\left(-D+E N_{s}^{(1)}\right) N_{s}^{(2)}\right) d s \\
& +k_{1} \int_{0}^{t}\left(\Pi_{s}\left(F N^{(1)}\right)-\Pi_{s}(F) \Pi_{s}\left(N_{s}^{(1)}\right)\right) d \nu_{s}^{1} \\
& +k_{2} \int_{0}^{t}\left(\Pi_{s}\left(F N_{s}^{(2)}\right)-\Pi_{s}(F) \Pi_{s}\left(N_{s}^{(2)}\right)\right) d \nu_{s}^{2}
\end{aligned}
$$

\section{The UNNORMALISED FILTERING EQUATION}

There are two difficulties with equation(3.1). Firstly, it contains the nonlinear terms $\Pi_{s}(F) \Pi_{s}\left(N^{(1)}\right), \Pi_{\bullet}(F) \Pi_{s}\left(N^{(2)}\right)$ and secondly, it is driven by the innovations processes $\nu_{t}^{1}, \nu_{t}^{2}$. Let the process be defined on $[0, T]$. The first step is to define a new measure $\bar{P}$ on the measurable space $(\Omega, \mathfrak{F})$ by

$$
\bar{P}(A)=\int \frac{d \bar{P}}{d P}(\omega) P(d \omega)
$$

for all $A \in \mathfrak{F}$, where

$$
\begin{aligned}
\left.\frac{d \bar{P}}{d P}\right|_{\mathfrak{F}_{1}} & =\Lambda_{t}^{-1}, \\
\mathfrak{F}_{t} & =\sigma\left\{y_{s}^{1}, y_{s}^{2}, N_{s}^{(1)}, N_{s}^{(2)}, s \leqslant t\right\},
\end{aligned}
$$

and

$$
\Lambda_{t}=\exp \left[\left(\int_{0}^{t} k_{1} N_{\jmath}^{(1)} d y_{\imath}^{1}+\int_{0}^{t} k_{2} N_{s}^{(2)} d y_{\imath}^{2}\right)-\frac{1}{2} \int_{0}^{t}\left(\left(k_{1} N_{\jmath}^{(1)}\right)^{2}+\left(k_{2} N_{s}^{(2)}\right)^{2}\right) d s\right] .
$$

Girsanov's Theorem. Suppose we start with a filtered probability space $\left(\Omega, \mathfrak{F}, \mathfrak{F}_{t}, \bar{P}\right)$ such that

(i) $y_{t}^{1}, y_{t}^{2}$ are independent Brownian motions;

(ii) $N_{t}^{1}, N_{t}^{2}$ satisfy Equation 1.3. 
We can define $P$ by setting $\frac{d P}{d \bar{P}} \mid \mathfrak{F}_{t}=\Lambda_{t}$.

Under $P: y_{t}^{1} y_{t}^{2}$ are not Brownian motions; in fact

$$
\begin{aligned}
& y_{t}^{1}-\int_{0}^{t} k_{s}^{1} N_{s}^{1} d s=B_{t}^{1} \text { is a Brownian motion under } P, \\
& y_{t}^{2}-\int_{0}^{t} k_{s}^{2} N_{s}^{2} d s=B_{t}^{2} \text { is a Brownian motion under } P .
\end{aligned}
$$

Consider any function $F \in C_{0}^{2}\left(R^{2}\right)$ with compact support. Using the Ito differential rule, we have

$$
\begin{aligned}
F\left(N_{t}^{(1)}, N_{t}^{(2)}\right)=F( & \left.N_{0}^{(1)}, N_{0}^{(2)}\right)+\int_{0}^{t} \frac{\partial F}{\partial N^{(1)}} d N_{a}^{(1)} \\
& +\int_{0}^{t} \frac{\partial F}{\partial F^{(2)}} d N_{\imath}^{(2)}+\frac{1}{2} \int_{0}^{t} \varepsilon_{1}^{2} \frac{\partial^{2} F}{\partial N^{(1) 2}} d s+\frac{1}{2} \int_{0}^{t} \varepsilon_{2}^{2} \frac{\partial^{2} F}{\partial N^{(2) 2}} d s
\end{aligned}
$$

that is

(4.1)

$$
\begin{aligned}
F\left(N_{t}^{(1)}, N_{t}^{(2)}\right)= & F\left(N_{0}^{(1)}, N_{0}^{(2)}\right)+\int_{0}^{t} \frac{\partial F}{\partial N^{(1)}}\left(\left(A-B N_{s}^{(1)}-C N_{s}^{(2)}\right) N_{s}^{(1)}\right) d s \\
& +\int_{0}^{t} \frac{\partial F}{\partial N^{(2)}}\left(\left(-D+E N_{s}^{(1)}\right) N_{s}^{(2)}\right) d s+\int_{0}^{t} \varepsilon_{1} \frac{\partial F}{\partial N_{1}} d W_{s}^{1} \\
& +\int_{0}^{t} \varepsilon_{2} \frac{\partial F}{\partial N_{2}} d W_{s}^{2}+\frac{1}{2} \int_{0}^{t}\left(\varepsilon_{1}^{2} \frac{\partial^{2} F}{\partial N^{(1) 2}}\right) d s+\frac{1}{2} \int_{0}^{t}\left[\varepsilon_{2}^{2} \frac{\partial^{2} F}{\partial N^{(2)}}\right] d s
\end{aligned}
$$

Let $\mathfrak{Y}_{t}=\sigma\left\{y_{s}^{1}, y_{s}^{2}, s \leqslant t\right\}$. Then using a Bayes-type theorem [11],

$$
\Pi_{t}\left(F\left(N_{t}^{(1)}, N_{t}^{(2)}\right)\right) \stackrel{d}{=} E\left[F\left(N_{t}^{(1)}, N_{t}^{(2)}\right) \mid \mathfrak{Y}_{t}\right]=\frac{\bar{E}\left[\Lambda_{t} F\left(N_{t}^{(1)}, N_{t}^{(2)}\right) \mid \mathfrak{Y}_{t}\right]}{\bar{E}\left[\Lambda_{t} \mid \mathfrak{Y}_{t}\right]}
$$

where $\bar{E}$ is the expectation under $\bar{P}$. Consider the numerator

$$
\bar{E}\left[\Lambda_{t} F\left(N_{t}^{(1)}, N_{t}^{(2)} \mid \mathfrak{Y}_{t}\right)\right] \stackrel{d}{=} \sigma_{t}\left(F\left(N_{t}^{(1)}, N_{t}^{(2)}\right)\right),
$$

where $\sigma_{t}\left(F\left(N_{t}^{(1)}, N_{t}^{(2)}\right)\right)$ is an unnormalised conditional distribution and is a measurevalued process. Further, if $F\left(N_{t}^{(1)}, N_{t}^{(2)}\right)=1$, then

$$
\bar{E}\left(\Lambda_{t} \mid \mathfrak{Y}_{t}\right)=\sigma_{t}(1) \text {. }
$$


Hence

$$
\Pi_{t}(F)=\frac{\sigma_{t}(F)}{\sigma_{t}(1)}
$$

We first obtain a semimartingale expression for $\sigma_{t}(1)$. Using the Ito differential rule, we get

$$
d \Lambda_{t}=\Lambda_{t}\left(k_{1} N_{t}^{(1)} d y_{t}^{1}+k_{2} N_{t}^{(2)} d y_{t}^{2}\right)
$$

That is,

$$
\Lambda_{t}=1+\int_{0}^{t} k_{1} N_{s}^{(1)} \Lambda_{s} d y_{s}^{1}+\int_{0}^{t} k_{2} N^{(2)} \Lambda_{2} d y_{s}^{2}
$$

so that $\Lambda_{t}$ is a $\left(\mathfrak{F}_{t}, \bar{P}\right)$-martingale. Consequently, as in Theorem $2.1, \widehat{\Lambda}_{t}=\bar{E}\left[\Lambda_{t} \mid \mathfrak{Y}_{t}\right]$ is a $\mathfrak{Y}_{t}$-martingale. Since the process $\left\{y_{t}^{1}\right\}$ and $\left\{y_{t}^{2}\right\}$ are Brownian motions under $\bar{P}$, there must exist $\mathfrak{Y}_{t}$-adapted processes $\left\{\eta_{t}^{1}\right\}$ and $\left\{\eta_{t}^{2}\right\}$ such that

$$
\widehat{\Lambda}_{t}=1+\int_{0}^{t} \eta_{s}^{1} d s+\int_{0}^{t} \eta_{s}^{2} d s
$$

To determine $\eta_{a}^{1}$, consider, using equation (4.3),

$$
\begin{aligned}
& y_{t}^{1} \Lambda_{t}=\int_{0}^{t} \Lambda_{s} d y_{s}^{1}+\int_{0}^{t} y_{a}^{1} k_{1} N_{a}^{1} \Lambda_{s} d y_{s}^{1}+\int_{0}^{t} \Lambda_{s} k_{1} N_{s}^{1} d s \\
& y_{1}^{2} \Lambda_{t}=\int_{0}^{t} \Lambda_{s} d y_{s}^{2}+\int_{0}^{t} y_{s}^{2} k_{2} N_{a}^{2} \Lambda_{s} d y_{s}^{2}+\int_{0}^{t} \Lambda_{s} k_{2} N_{s}^{2} d s .
\end{aligned}
$$

Conditioning on $\mathfrak{Y}_{t}$ under measure $\bar{P}$,

$$
\begin{aligned}
& \bar{E}\left[y_{t}^{1} \Lambda_{t} \mid \mathfrak{Y}_{t}\right]=y_{t}^{1} \widehat{\Lambda}_{t}=\int_{0}^{t} k_{1} \Lambda_{s} N_{t}^{1} d s+M_{t}^{1} \\
& \bar{E}\left[y_{t}^{2} \Lambda_{t} \mid \mathfrak{Y}_{t}\right]=y_{t}^{2} \widehat{\Lambda}_{t}=\int_{0}^{t} k_{2} \Lambda_{,} N_{s}^{2} d s+M_{t}^{2}
\end{aligned}
$$

where $M_{t}^{1}$ and $M_{t}^{2}$ are $\left(\mathfrak{Y}_{t}, \bar{P}\right)$-martingales. However, from equation (4.5) and Ito's rule,

$$
\begin{aligned}
& y_{t}^{1} \widehat{\Lambda}_{t}=\int_{0}^{t} \eta_{s}^{1} d s+R_{t}^{1}, \\
& y_{1}^{2} \widehat{\Lambda}_{t}=\int_{0}^{t} \eta_{s}^{2} d s+R_{t}^{2} .
\end{aligned}
$$


The decompositions (4.8) and (4.10) must be the same, so too (4.9) and (4.11). Hence

$$
M_{t}^{1}=R_{t}^{1} \quad \text { and } \quad M_{t}^{2}=R_{t}^{2}
$$

which implies that

$$
\begin{aligned}
& \eta_{s}^{1}=k_{1} \Lambda_{s} N_{s}^{(1)}=k_{1} \bar{E}\left[\Lambda_{s} N_{s}^{(1)} \mid \mathfrak{Y}_{s}\right] \\
& \eta_{s}^{2}=k_{2} \Lambda_{s} N_{s}^{(2)}=k_{2} \bar{E}\left[\Lambda_{s} N_{s}^{(2)} \mid \mathfrak{Y}_{s}\right]
\end{aligned}
$$

Using Bayes' rule again, we see that

$$
\begin{aligned}
& \eta_{s}^{1}=k_{1} \widehat{\Lambda}_{s} \Pi_{s}\left(N_{s}^{1}\right), \\
& \eta_{s}^{2}=k_{2} \widehat{\Lambda}_{s} \Pi_{s}\left(N_{s}^{2}\right),
\end{aligned}
$$

Substituting for $\eta_{s}^{1}$ and $\eta_{s}^{2}$ in equation (4.5) we get

$$
\widehat{\Lambda}_{t}=1+\int_{0}^{t} k_{1} \widehat{\Lambda}_{s} \Pi_{s}\left(N_{s}^{(1)}\right) d s+\int_{0}^{t} k_{2} \widehat{\Lambda}_{s} \Pi_{s}\left(N_{s}^{(2)}\right) d s
$$

However, equation (4.12) has the unique solution

$$
\begin{aligned}
\hat{\Lambda}_{t}= & \exp \left(\int_{0}^{t} k_{1} \Pi_{s}\left(N_{s}^{(1)}\right) d y_{s}^{1}+\int_{0}^{t} k_{2} \Pi_{s}\left(N_{s}^{(2)}\right) d y_{s}^{2}\right. \\
& \left.\quad-\frac{1}{2} \int_{0}^{t}\left(k_{1}^{2} \Pi_{s}\left(N_{s}^{(1)}\right)^{2}+k_{2}^{2} \Pi_{2}\left(N_{s}^{(2)}\right)^{2}\right) d s\right) \\
= & \sigma_{t}(1) .
\end{aligned}
$$

(Note that ${ }^{-}$denotes conditioning under measure $\bar{P}$, while $I$ denotes conditioning under the original measure $P$.)

From the equations (4.1) and (4.2), using Ito's rule, we get

$$
\begin{aligned}
& F\left(N_{t}^{(1)}, N_{t}^{(2)}\right) \Lambda_{t}=F\left(N_{0}^{(1)}, N_{0}^{(2)}\right)+\int_{0}^{t} \Lambda_{s} \frac{\partial F}{\partial N_{0}^{(1)}}\left(\left(A-B N_{a}^{(1)}-C N_{a}^{(2)}\right) N_{a}^{(1)}\right) d s \\
& +\int_{0}^{t} \Lambda_{s} \frac{\partial F}{\partial N^{(1)}} \varepsilon_{1} d W_{s}^{1}+\int_{0}^{t} \Lambda_{0} \frac{\partial F}{\partial N^{(2)}}\left(\left(-D+E N_{a}^{(1)}\right) N_{a}^{(2)}\right) d s+\int_{0}^{t} \Lambda_{\iota} \frac{\partial F}{\partial N^{(2)}} \varepsilon_{2} d W_{s}^{2} \\
& +\frac{1}{2} \int_{0}^{t} \Lambda_{\bullet}\left(\varepsilon_{1}^{2} \frac{\partial^{2} F}{\partial N^{(1)^{2}}}+\varepsilon_{2}^{2} \frac{\partial^{2} F}{\partial N^{(2)^{2}}}\right) d s+\int_{0}^{t} \Lambda_{\triangleleft} F_{\bullet}\left(k_{1} N_{s}^{(1)} d y_{s}^{1}+k_{2} N_{s}^{(2)} d y_{\triangleleft}^{2}\right) .
\end{aligned}
$$


Conditioning each side on $\mathfrak{Y}_{t}$ under measure $\bar{P}$, we have

$$
\begin{aligned}
\bar{E} & {\left[\Lambda_{t} F\left(N_{t}^{(1)}, N_{t}^{(2)}\right) \mid \mathfrak{Y}_{t}\right]=\bar{E}\left[F\left(N_{0}^{(1)}, N_{0}^{(2)}\right) \mid \mathfrak{Y}_{t}\right] } \\
& +\bar{E}\left[\left(\int_{0}^{t} \Lambda_{s} \frac{\partial F}{\partial N^{(1)}}\left(\left(A-B N_{s}^{(1)}-C N_{s}^{(2)}\right) N_{s}^{(1)}\right) d s\right.\right. \\
& +\int_{0}^{t} \Lambda_{s} \frac{\partial F}{\partial N^{(1)}} \varepsilon_{1} d W_{s}^{1}+\int_{0}^{t} \Lambda_{s} \frac{\partial F}{\partial N^{(2)}}\left(\left(-D+E N_{s}^{(1)}\right) N_{s}^{(2)}\right) d s+\int_{0}^{t} \Lambda_{s} \frac{\partial F}{\partial N^{(2)}} \varepsilon_{2} d W_{s}^{2} \\
& \left.\left.+\frac{1}{2} \int_{0}^{t} \Lambda_{s}\left(\varepsilon_{1}^{2} \frac{\partial^{2} F}{\partial N^{(1)^{2}}}+\varepsilon_{2}^{2} \frac{\partial^{2} F}{\partial N^{(2)^{2}}}\right) d s+\int_{0}^{t} \Lambda_{s} F_{s}\left(k_{1} N_{s}^{1} d y_{s}^{1}+k_{2} N_{s}^{2} d y_{s}^{2}\right)\right) \mid \mathfrak{Y}_{t}\right] \\
& =\bar{E}\left[F\left(N_{0}^{(1)}, N_{0}^{(2)}\right) \mid \mathfrak{Y}_{1}\right]+\int_{0}^{t} \bar{E}\left[\Lambda_{s} \frac{\partial F}{\partial N^{(1)}}\left(\left(A-B N_{s}^{(1)}-C N_{s}^{(2)}\right) N_{s}^{(1)}\right) \mid \mathfrak{Y}_{s}\right] d s \\
& +\int_{0}^{t} \bar{E}\left[\Lambda_{s} \frac{\partial F}{\partial F^{(2)}}\left(\left(-D+E N_{s}^{(1)}\right) N_{s}^{(2)}\right) \mid \mathfrak{Y}_{t}\right] d s \\
& +\frac{1}{2} \int_{0}^{t} \bar{E}\left[\Lambda_{s}\left(\varepsilon_{1}^{2} \frac{\partial^{2} F}{\partial N_{s}^{(1)^{2}}}+\varepsilon_{2}^{1} \frac{\partial^{2} F}{\left.\partial N^{(2)}\right)^{2}}\right) \mid \mathfrak{Y}_{s}\right] d s \\
& +\int_{0}^{t} \bar{E}\left[\Lambda_{s} F_{s} k_{1} N_{s}^{(1)} \mid \mathfrak{Y}_{s}\right] d y_{s}^{1}+\int_{0}^{t} \bar{E}\left[\Lambda_{s} F_{s} k_{1} N_{s}^{(2)} \mid \mathfrak{Y}_{s}\right] d y_{s}^{2} .
\end{aligned}
$$

As a result, we have the Zakai equation for the unnormalised conditional distribution:

$$
\begin{aligned}
\sigma_{t}\left(F\left(N_{t}^{(1)}, N_{t}^{(2)}\right)\right)= & \sigma_{0}\left(F\left(N_{0}^{(1)}, N_{0}^{(2)}\right)\right) \\
& +\int_{0}^{t} \sigma_{s}\left(\frac{\partial F}{\partial N^{(1)}}\left(N_{s}^{(1)}\left(A-B N_{s}^{(1)}-C N_{s}^{(2)}\right)\right)\right) d s \\
& +\int_{0}^{t} \sigma_{s}\left(\frac{\partial F}{\partial N^{(2)}}\left(N_{s}^{(2)}\left(-D+E N_{s}^{(1)}\right)\right)\right) d s \\
& +\frac{\varepsilon_{1}^{2}}{2} \int_{0}^{t} \sigma_{s}\left(\frac{\partial^{2} F}{\partial N^{(1)^{2}}}\right) d s+\frac{\varepsilon_{2}^{2}}{2} \int_{0}^{t} \sigma_{s}\left(\frac{\partial^{2} F}{\partial N^{(2)^{2}}}\right) d s \\
& +k_{1} \int_{0}^{t} \sigma_{s}\left(F_{s} N_{s}^{(1)}\right) d y_{s}^{1}+k_{2} \int_{0}^{t} \sigma_{s}\left(F_{s} N_{s}^{(2)}\right) d y_{s}^{2}
\end{aligned}
$$

\section{Decomposition of SOlution}

The equation (4.16) contains stochastic integrals. We now apply the decomposition techniques of Kunita [6, Section 5.2] to obtain an expression that involves no stochastic integrals. 
In terms of Stratonovich integrals [10], Equation (4.16) becomes

$$
\begin{aligned}
& \sigma_{t}\left(F\left(N_{t}^{(1)}, N_{t}^{(2)}\right)\right)=\sigma_{0}\left(F\left(N_{0}^{(1)}, N_{0}^{(2)}\right)\right) \\
& \quad+\int_{0}^{t} \sigma_{s}\left(A F\left(N_{s}^{(1)}, N_{s}^{(2)}\right)-\frac{k_{1}^{2}}{2}\left(N_{s}^{(1)}\right)^{2} F\left(N_{s}^{(1)}, N_{s}^{(2)}\right)\right. \\
& \left.\quad-\frac{k_{2}^{2}}{2}\left(N_{s}^{(2)}\right)^{2} F\left(N_{s}^{(1)}, N_{s}^{(2)}\right)\right) d s, \\
& \quad+k_{1} \int_{0}^{t} \sigma_{s}\left(N_{s}^{(1)} F\left(N_{s}^{(1)}\right)\right) \circ d y_{s}^{1}+k_{2} \int_{0}^{t} \sigma_{s}\left(N_{s}^{(2)} F\left(N_{s}^{(2)}\right)\right) \circ d y_{s}^{2} .
\end{aligned}
$$

where

$$
\begin{aligned}
A F\left(N_{s}^{(1)},\right. & \left.N_{s}^{(2)}\right)=\left(N_{s}^{(1)}\left(A-B N_{a}^{(1)}-C N_{s}^{(2)}\right)\right) \frac{\partial F}{\partial N^{(1)}}\left(N_{s}^{(1)}, N_{s}^{(2)}\right) \\
& +\left(N_{s}^{(2)}\left(-D+E N_{a}^{(1)}\right)\right) \frac{\partial F}{\partial N^{(2)}}\left(N_{a}^{(1)}, N_{s}^{(2)}\right)+\frac{\varepsilon_{1}^{2}}{2} \frac{\partial^{2} F}{\partial N^{(1)^{2}}}+\frac{\varepsilon_{2}^{2}}{2} \frac{\partial^{2} F}{\partial N^{(2)}} .
\end{aligned}
$$

Consider the following operators defined on the functons $F\left(N_{1}^{(1)}, N^{(2)}\right)$ :

$$
\begin{aligned}
L(t) F\left(N^{(1)}, N^{(2)}\right)= & A F\left(N^{(1)}, N^{(2)}\right)-\frac{k_{1}^{2}}{2}\left(N^{(1)}\right)^{2} F\left(N^{(1)}, N^{(2)}\right) \\
& -\frac{k_{2}^{2}}{2}\left(N^{(2)}\right)^{2} F\left(N^{(1)}, N^{(2)}\right) \\
\mu_{t} F\left(N^{(1)}, N^{(2)}\right)= & F\left(N^{(1)}, N^{(2)}\right) \exp \left(-\sum_{i=1}^{2} k_{i} N^{(i)} y_{t}^{i}\right) \\
\mu_{t}^{-1} F\left(N^{(1)}, N^{(2)}\right)= & F\left(N^{(1)}, N^{(2)}\right) \exp \left(-\sum_{i=1}^{2} k_{i} N^{(i)} y_{t}^{(i)}\right) .
\end{aligned}
$$

Denoting

$$
\begin{aligned}
& \Delta\left(N^{(1)}\right)=\left(A-B N^{(1)}-C N^{(2)}\right) N^{(1)}, \\
& \Delta\left(N^{(2)}\right)=\left(-D+E N^{(1)}\right) N^{(2)}
\end{aligned}
$$

we have

$$
\begin{array}{r}
{\left[\mu_{t} L(t) \mu_{t}^{-1}\right] F\left(N^{(1)}, N^{(2)}\right)=\sum_{i=1}^{2}\left\{\frac{\varepsilon_{1}^{2}}{2} \frac{\partial^{2} F}{\partial N^{(1)^{2}}}+\left(\left(\Delta\left(N^{(i)}\right)-k_{i} \varepsilon_{i}^{2} y_{i}^{i}\right) \frac{\partial F}{\partial N^{(i)}}\right)\right.} \\
\left.+\left(\frac{k_{i}^{2} \varepsilon_{i}^{2}\left(y_{t}^{i}\right)^{2}}{2}-\Delta\left(N^{(i)}\right) y_{t}^{i}-\frac{k_{i}^{2}\left(N^{(i)}\right)^{2}}{2}\right) F\left(N^{(1)}, N^{(2)}\right)\right\}
\end{array}
$$


Let $\left(\tilde{N}_{t}^{(1)}, \tilde{N}_{t}^{(2)}\right)$ be the solution of the system

$$
\tilde{N}_{t}^{(i)}=\tilde{N}_{0}^{(i)}+\int_{0}^{t}\left(\Delta\left(N_{s}^{(i)}-k_{i} \varepsilon_{i}^{2} y_{\iota}^{i}\right) d s+\varepsilon_{i} W_{t}^{i}\right), \quad i=1,2 .
$$

Considering the expectation, given $\mathfrak{Y}_{t}$, of

$$
F\left(\tilde{N}_{t}^{(1)}, \tilde{N}_{t}^{(2)}\right) \exp \left\{\int_{0}^{t} \sum_{i=1}^{2}\left(\frac{\left(\varepsilon_{i} y^{i} k_{i}\right)^{2}}{2}-\Delta\left(\tilde{N}_{a}^{(i)}\right) y_{a}^{i}-\frac{k_{i}^{2}}{2}\left(\tilde{N}_{a}^{(i)}\right)^{2}\right) d s\right\}
$$

and letting

$$
\begin{aligned}
\nu_{t}(F)=E & \left(F\left(\tilde{N}_{t}^{(1)}, \tilde{N}_{i}^{(2)}\right)\right. \\
& \left.\times \exp \left\{\int_{0}^{t} \sum_{i=1}^{2}\left(\frac{\left(\varepsilon_{i} k_{i} y_{s}^{i}\right)^{2}}{2}-\Delta\left(\widehat{N}_{s}^{(i)}\right) y_{s}^{(i)}-\frac{k_{1}^{2}}{2}\left(\widehat{N}_{s}^{(i)}\right)^{2}\right) d s\right\} \mid \mathfrak{Y}_{t}\right)
\end{aligned}
$$

where the expectation is taken over the forward sample paths, we have

$$
\nu_{t}(F)=V_{0}(F)+\int_{0}^{t} \nu_{s}\left(\mu_{s} L(s) \mu_{s}^{-1}\right) F\left(N_{s}^{(1)}, N_{s}^{(2)}\right) d s
$$

that is, $\nu_{t}(F)$ solves equation (5.4). Applying Kunita's Theorem, the solution of the Zakai equation is given by

$$
\sigma_{t}(F)=\nu_{t}\left(\mu_{t} F\right)(x)
$$

Evaluating $\nu_{t}\left(\mu_{t} F\right)$, we have

$$
\begin{aligned}
& \text { (5.9) } \nu_{t}\left(\mu_{t} F\right)=\nu_{0}(F)+\int_{0}^{t} \nu_{s}\left(\mu_{s} L(s) \mu_{s}^{-1}\right)\left(\mu_{s} F\right) d s \\
& +k_{1} \int_{0}^{t} \nu_{0}\left(\mu_{s}\left(N_{s}^{(1)} F\left(N_{s}^{(1)}, N_{s}^{(2)}\right)\right)\right) \circ d y_{s}^{1}+k_{2} \int_{0}^{t} \nu_{s}\left(\mu_{s}\left(N_{s}^{(2)} F\left(N_{s}^{(1)}, N_{s}^{(2)}\right)\right)\right) \circ d y_{s}^{2} .
\end{aligned}
$$

Hence, the solution of the Zakai equation for the unnormalised conditional distribution is given by

$$
\begin{aligned}
& \sigma_{t}(F)=\nu_{t}\left(\mu_{a} F\right)=E\left\{\left(\exp \sum_{i=1}^{2} k_{i} \widehat{N}^{(i)} y_{t}^{i}\right) F\left(\tilde{N}_{t}^{(1)}, N_{t}^{(2)}\right)\right. \\
&\left.\times \exp \left\{\int_{0}^{t} \sum_{i=1}^{2}\left(\frac{\left(\varepsilon_{i} k_{i} y_{i}\right)^{2}}{2} \Delta\left(\tilde{N}^{(i)}\right) y^{i}-\frac{k_{i}^{2}}{2}\left(\tilde{N}^{(i)}\right)^{2}\right) d s\right\} \mid \mathfrak{Y}_{t}\right\}
\end{aligned}
$$

The advantage of this expression (5.10) over (4.16) is that it involves no stochastic integrals. The observation trajectories $y_{i}^{i}, i=1,2$, appear just as parameters. Further, the operator $\mu_{t} L(t) \mu_{t}^{-1}$ differs from $L(t)$ only by terms of less than second order. 


\section{ZAKAI EQUATION FOR UNNORMALISED CONDITIONAL DENSITY}

Now, suppose that $\sigma_{t}$ has a density $q_{t}\left(N^{(1)}, N^{(2)}\right)$. We then have

$$
\begin{aligned}
\sigma_{t} & \left(F\left(N_{t}^{(1)}, N_{t}^{(2)}\right)\right)=\int_{\mathscr{R}^{2}} F\left(N^{(1)}, N^{(2)}\right) q_{t}\left(N^{(1)}, N^{(2)}\right) d N^{(1)} d N^{(2)} \\
& =\int_{\mathfrak{R}_{2}} F\left(N^{(1)}, N^{(2)}\right) q_{0}\left(N^{(1)}, N^{(2)}\right) d N^{(1)} d N^{(2)} \\
& +\int_{0}^{t}\left(\int_{\mathfrak{R}^{2}} \frac{\partial F}{\partial N^{(1)}}\left(N^{(1)}, N^{(2)}\right)\left(N^{(1)}\left(A-B N^{(1)}-C N^{(2)}\right)\right) q_{t}\left(N^{(1)}, N^{(2)}\right) d N^{(1)} d N^{(2)}\right) d s \\
& +\int_{0}^{t}\left(\int_{\mathscr{R}^{2}} \frac{\partial F}{\partial N^{(2)}}\left(N^{(1)}, N^{(2)}\right)\left(N^{(2)}\left(-D+E N^{(1)}\right)\right) q_{t}\left(N^{(1)}, N^{(2)}\right) d N^{(1)} d N^{(2)}\right) d s \\
& +\frac{\varepsilon_{1}^{2}}{2} \int_{0}^{t}\left(\int_{\mathfrak{R}^{2}}\left(\frac{\partial^{2} F}{\partial N^{(1)^{2}}}\left(N^{(1)}, N^{(2)}\right)\right) q_{t}\left(N^{(1)}, N^{(2)}\right) d N^{(1)} d N^{(2)}\right) d s \\
& +\frac{\varepsilon_{2}^{2}}{2} \int_{0}^{t}\left(\int_{\mathfrak{R}^{2}}\left(\frac{\partial^{2} F}{\partial N^{(2)^{2}}}\left(N^{(1)}, N^{(2)}\right)\right) q_{t}\left(N^{(1)}, N^{(2)}\right) d N^{(1)} d N^{(2)}\right) d s .
\end{aligned}
$$

Since $F \in C_{0}^{2}\left(\Re^{2}\right)$ with compact support, integrating by parts yields

$$
\begin{aligned}
\int_{\mathfrak{R}^{2}} & \frac{\partial F}{\partial N^{(1)}}\left(N^{(1)}, N^{(2)}\right)\left(N^{(1)}\left(A-B N^{(1)}-C N^{(2)}\right)\right) q_{s}\left(N^{(1)}, N^{(2)}\right) d N^{(1)} d N^{(2)} \\
= & \left.F\left(N^{(1)}, N^{(2)}\right)\left(N^{(1)}\left(A-B N^{(1)}-C N^{(2)}\right)\right) q_{s}\left(N^{(1)}, N^{(2)}\right)\right|_{-\infty} ^{\infty} \\
& -\int_{\mathfrak{R}^{2}} F\left(N^{(1)}, N^{(2)}\right) \frac{\partial}{\partial N^{(1)}}\left(N^{(1)}\left(A-B N^{(1)}-C N^{(2)}\right) q_{s}\left(N^{(1)}, N^{(2)}\right)\right) d N^{(1)} d N^{(2)} \\
= & -\int_{\mathfrak{R}^{2}} F\left(N^{(1)}, N^{(2)}\right)\left\{\left(A-2 B N^{(1)}-C N^{(2)}\right) q_{\bullet}\left(N^{(1)}, N^{(2)}\right)\right. \\
& \left.+\left(N^{(1)}\left(A-B N^{(1)}-C N^{(2)}\right)\right) \frac{\partial q_{s}}{\partial N^{(1)}}\left(N^{(1)}, N^{(2)}\right)\right\} d N^{(1)} d N^{(2)} \\
\int_{\mathfrak{R}^{2}} & \frac{\partial F}{\partial N^{(2)}}\left(N^{(1)}, N^{(2)}\right)\left(N^{(2)}\left(-D+E N^{(1)}\right)\right) q_{s}\left(N^{(1)}, N^{(2)}\right) d N^{(1)} d N^{(2)} \\
= & \left.F\left(N^{(1)}, N^{(2)}\right)\left(N^{(2)}\left(-D+E N^{(1)}\right)\right) q_{s}\left(N^{(1)}, N^{(2)}\right)\right|_{-\infty} ^{\infty} \\
& -\int_{\mathfrak{R}^{2}} F\left(N^{(1)}, N^{(2)}\right)\left\{\left(-D+E N^{(1)}\right) q_{s}\left(N^{(1)}, N^{(2)}\right)\right. \\
& \left.+\left(N^{(2)}\left(-D+E N^{(1)}\right)\right) \frac{\partial q_{s}}{\partial N^{(2)}}\left(N^{(1)}, N^{(2)}\right)\right\} d N^{(1)} d N^{(2)} \\
= & -\int_{\mathscr{R}^{2}} F\left(N^{(1)}, N^{(2)}\right)\left\{\left(-D+E N^{(1)}\right) q_{s}\left(N^{(1)}, N^{(2)}\right)\right. \\
& \left.+\left(N^{(2)}\left(-D+E N^{(1)}\right)\right) \frac{\partial q_{s}}{\partial N^{(2)}}\left(N^{(1)}, N^{(2)}\right)\right\} d N^{(1)} d N^{(2)} \\
&
\end{aligned}
$$




$$
\begin{aligned}
& \int_{\mathfrak{R}^{2}} \frac{\partial^{2} F}{\partial N^{(1)^{2}}}\left(N^{(1)}, N^{(2)}\right) q_{\star}\left(N^{(1)}, N^{(2)}\right) d N^{(1)} d N^{(2)} \\
& \quad=\int_{\mathfrak{R}^{2}} F\left(N^{(1)}, N^{(2)}\right) \frac{\partial^{2} q_{s}}{\partial N^{(1)^{2}}}\left(N^{(1)}, N^{(2)}\right) d N^{(1)} d N^{(2)} \\
& \int_{\mathfrak{R}^{2}} \frac{\partial^{2} F}{\partial N^{(2)^{2}}}\left(N^{(1)}, N^{(2)}\right) q_{s}\left(N^{(1)}, N^{(2)}\right) d N^{(1)} d N^{(2)} \\
& \quad=\int_{\mathfrak{R}^{2}} F\left(N^{(1)}, N^{(2)}\right) \frac{\partial^{2} q_{s}}{\partial N^{(2)^{2}}}\left(N^{(1)}, N^{(2)}\right) d N^{(1)} d N^{(2)} .
\end{aligned}
$$

Substituting in equation (6.1) gives

$$
\begin{aligned}
& \int_{\mathfrak{R}^{2}} F\left(N^{(1)}, N^{(2)}\right) q_{t}\left(N^{(1)}, N^{(2)}\right) d N^{(1)} d N^{(2)} \\
& =\int_{\mathfrak{R}^{2}} F\left(N^{(1)}, N^{(2)}\right) q_{0}\left(N^{(1)}, N^{(2)}\right) d N^{(1)} d N^{(2)} \\
& +\int_{0}^{t}\left(\int _ { \mathfrak { R } ^ { 2 } } F ( N ^ { ( 1 ) } , N ^ { ( 2 ) } ) \left(\left(-A+2 B N^{(1)}+C N^{(2)}\right) q_{d}\left(N^{(1)}, n^{(2)}\right)\right.\right. \\
& \left.\left.+\left(N^{(1)}\left(-A+B N^{(1)}+C N^{(2)}\right)\right) \frac{\partial q_{d}}{\partial N^{(1)}}\left(N^{(1)}, N^{(2)}\right)\right) d N^{(1)} d N^{(2)}\right) d s \\
& +\int_{0}^{t}\left\{\int _ { \mathfrak { R } ^ { 2 } } F ( N ^ { ( 1 ) } , N ^ { ( 2 ) } ) \left(\left(D-E N^{(1)}\right) q_{\mathcal{J}}\left(N^{(1)}, N^{(2)}\right)\right.\right. \\
& \left.\left.+\left(N^{(2)}\left(D-E N^{(1)}\right)\right) \frac{\partial q_{s}}{\partial N^{(2)}}\left(N^{(1)}, N^{(2)}\right)\right)\right\} \times\left(d N^{(1)} D N^{(2)}\right) d s \\
& +\frac{\alpha_{1}^{2}}{2} \int_{0}^{t}\left(\int_{\mathscr{R}^{2}} F\left(N^{(1)}, N^{(2)}\right) \frac{\partial^{2} q s}{\partial N^{(1)^{2}}} d N^{(1)} d N^{(2)}\right) d s \\
& +\frac{\epsilon_{2}^{2}}{2} \int_{0}^{t}\left(\int_{\mathscr{R}^{2}} F\left(N^{(1)}, N^{(2)}\right) \frac{\partial^{2} q_{s}}{\partial N^{(2)^{2}}} d N^{(1)} d n^{(2)}\right) d s \\
& +k_{1} \int_{0}^{t}\left(\int_{\mathfrak{R}^{2}}\left(F\left(N^{(1)}, N^{(2)}\right) N^{(1)}\right) q_{\perp}\left(N^{(1)}, N^{(2)}\right) d N^{(1)} d N^{(2)}\right) d y^{1} \\
& +k_{2} \int_{0}^{t}\left(\int_{\Re^{2}}\left(F\left(N^{(1)}, N^{(2)}\right) N^{(2)}\right) q_{s}\left(N^{(1)}, N^{(2)}\right) d N^{(1)} d N^{(2)}\right) d y_{8}^{2} .
\end{aligned}
$$

Hence

$$
\begin{aligned}
\sigma^{t}( & \left.F\left(N_{t}^{(1)}, N_{t}^{(2)}\right)\right)=\bar{E}\left[\Lambda_{t} F\left(N_{t}^{(1)}, N_{t}^{(2)}\right)\right]=\left\langle F, q_{t}\right\rangle \\
& +\int_{\mathfrak{R}^{2}} F\left(N^{(1)}, N^{(2)}\right) q_{t}\left(N^{(1)}, N^{(2)}\right) d N^{(1)} d N^{(2)} \\
= & \int_{\mathfrak{R}^{2}} F\left(N^{(1)}, N^{(2)}\right) q_{0}\left(N^{(1)}, N^{(2)}\right) d N^{(1)} d N^{(2)} \\
& +\int_{\mathfrak{R}^{2}} F\left(N^{(1)}, N^{(2)}\right)\left\{\int _ { 0 } ^ { t } \left(\left(-A+2 B N^{(1)}+C N^{(2)}\right) q_{s}\left(N^{(1)}, N^{(2)}\right)\right.\right.
\end{aligned}
$$




$$
\begin{aligned}
& \left.\left.+\left(N^{(1)}\left(-A+B N^{(1)}+C N^{(2)}\right)\right) \frac{\partial q_{s}}{\partial N^{(1)}}\left(N^{(1)}, N^{(2)}\right)\right) d s\right\} d N^{(1)} d N^{(2)} \\
& +\int_{\mathfrak{R}^{2}} F\left(N^{(1)}, N^{(2)}\right)\left\{\int _ { 0 } ^ { t } \left(\left(D-E N^{(1)}\right) q_{\bullet}\left(N^{(1)}, N^{(2)}\right)\right.\right. \\
& \left.\left.+\left(N^{(2)}\left(D-E N^{(1)}\right)\right) \frac{\partial q_{s}}{\partial N^{(2)}}\left(N^{(1)}, N^{(2)}\right)\right) d s\right\} \times d N^{(1)} d N^{(2)} \\
& +\int_{\mathfrak{R}^{2}} F\left(N^{(1)}, N^{(2)}\right)\left(\frac{e_{1}^{2}}{2} \int_{0}^{t} \frac{\partial^{2} q_{s} d s}{\partial N^{(1)^{2}}}\right) d N^{(1)} d N^{(2)} \\
& +\int_{\mathfrak{R}^{2}} F\left(N^{(1)}, N^{(2)}\right)\left(\frac{e_{2}^{2}}{2} \int_{0}^{t} \frac{\partial^{2} q_{s} d s}{\partial N^{(2)^{2}}}\right) d N^{(1)} d N^{(2)} \\
& +\int_{\mathfrak{R}^{2}} F\left(N^{(1)}, N^{(2)}\right)\left(k_{1} \int_{0}^{t} N^{(1)} q_{s}\left(N^{(1)}, N^{(2)}\right) d y_{s}^{1}\right) d N^{(1)} d N^{(2)} \\
& +\int_{\mathfrak{R}^{2}} F\left(N^{(1)}, N^{(2)}\right)\left(k_{2} \int_{0}^{t} N^{(2)} q_{s}\left(N^{(1)}, N^{(2)}\right) d y_{s}^{2}\right) d N^{(1)} d N^{(2)}
\end{aligned}
$$

Hence, the adjoint Zakai equation for the unnormalised conditional density $q_{t}\left(N^{(1)}, N^{(2)}\right)$ given $\mathfrak{Y}_{t}=\sigma\left\{y_{s}^{1}, y_{s}^{2} ; s \leqslant t\right\}$ is given by

$$
\begin{aligned}
& q_{t}\left(N^{(1)}, N^{(2)}\right)=q_{0}\left(N^{(1)}, N^{(2)}\right)+\int_{0}^{t}\left[\left(-A+2 B N^{(1)}+C N^{(2)}\right) q_{s}\left(N^{(1)}, N^{(2)}\right)\right. \\
& \left.+\left(N^{(1)}\left(-A+B N^{(1)}+C N^{(2)}\right)\right) \frac{\partial q_{s}}{\partial N^{(1)}}\left(N^{(1)}, N^{(2)}\right)\right] d s \\
& +\left[\int_{0}^{t}\left(D-E N^{(1)}\right) q_{s}\left(N^{(1)}, N^{(2)}\right)+\left(N^{(2)}\left(D-E N^{(1)}\right)\right) \frac{\partial q_{s}}{\partial N^{(2)}}\left(N^{(1)}, N^{(2)}\right)\right] d s \\
& +\frac{\varepsilon_{1}^{2}}{s} \int_{0}^{t} \frac{\partial^{2} q_{s}}{\partial N^{(1)^{2}}} d s+\frac{\varepsilon_{2}^{2}}{2} \int_{0}^{t} \frac{\partial^{2} q_{s}}{\partial N^{(2)^{2}}} d s \\
& +k_{1} \int_{0}^{t} N^{(1)} q_{s}\left(N^{(1)}, N^{(2)}\right) d y^{1}+k_{2} \int_{0}^{t} N^{(2)} q_{s}\left(N^{(1)}, N^{(2)}\right) d y^{2}
\end{aligned}
$$

The above expression again contains stochastic integrals. We once more apply the decomposition techniques of Kunita [6, Section 5.2] to enable us to obtain a density free of such integrals. The approach is similar to that adopted for the Zakai equation.

Let

$$
\begin{aligned}
& A^{*} q\left(N^{(1)}, N^{(2)}\right)=-\frac{\partial}{\partial N^{(1)}}\left[\left(A-B N^{(2)}-C N^{(2)}\right) N^{(1)} q\left(N^{(1)}, N^{(2)}\right)\right] \\
& \quad-\frac{\partial}{\partial N^{(2)}}\left[\left(-D+E N^{(1)}\right) N^{(2)} q\left(N^{(1)}, N^{(2)}\right)\right]+\frac{\varepsilon_{1}^{2}}{2} \frac{\partial^{2} q}{\partial N^{(1)^{2}}}+\frac{\varepsilon_{2}^{2}}{2} \frac{\partial^{2} q}{\partial N^{(2)^{2}}}
\end{aligned}
$$


The equation (6.2) takes the form

$$
\begin{aligned}
q_{t}\left(N^{(1)}, N^{(2)}\right)= & q_{0}\left(N^{(1)}, N^{(2)}\right)+\int_{0}^{t} A^{*} q_{s}\left(N^{(1)}, N^{(2)}\right) d s \\
& +k_{1} \int_{0}^{t} N^{(1)} q_{s}\left(N^{(1)}, N^{(2)}\right) d y_{s}^{1}+k_{2} \int_{0}^{t} N^{(2)} q_{s}\left(N^{(1)}, N^{(2)}\right) d y_{s}^{2},
\end{aligned}
$$

where $A^{*}$ is the adjoint of $A$. In terms of Stratonovich integrals, this becomes

$$
\begin{aligned}
& \text { (6.11) } q_{t}\left(N^{(1)}, N^{(2)}\right)=q_{0}\left(N^{(1)}, N^{(2)}\right)+\int_{0}^{t}\left(A^{*}-\frac{k_{1}^{2}}{x}\left(N^{(1)}\right)^{2}-\frac{k_{2}^{2}}{2}\left(N^{(2)}\right)^{2}\right) \\
& \times q_{s}\left(N^{(1)}, N^{(2)}\right) d s+k_{1} \int_{0}^{t} N^{(1)} q_{s}\left(N^{(1)}, N^{(2)}\right) \circ d y_{s}^{1}+k_{2} \int_{0}^{t} N^{(2)} q_{s}\left(N^{(1)}, N^{(2)}\right) \circ d y_{s}^{2} .
\end{aligned}
$$

Consider the following operators defined on $q\left(N^{(1)}, N^{(2)}\right)$ :

$$
\begin{gathered}
L^{*}(t) q\left(N^{(1)}, N^{(2)}\right)=A^{*} q\left(N^{(1)}, N^{(2)}\right)-\frac{k_{1}^{2}}{2}\left(N^{(1)}\right)^{2} q\left(N^{(1)}, N^{(2)}\right) \\
-\frac{k_{2}^{2}}{2}\left(N^{(2)}\right)^{2} q\left(N^{(1)}, N^{(2)}\right), \\
\mu_{t} q\left(N^{(1)}, N^{(2)}\right)= \\
\mu_{t}^{-1} q\left(N^{(1)}, N^{(2)}\right) \cdot \exp \left(\sum_{i=1}^{2} k_{i} N^{(i)} y_{t}^{(i)},\right. \\
\end{gathered}
$$

We then obtain

(6.14)

$$
\begin{aligned}
& \left(\mu_{t}^{-1} L^{*}(t) \mu_{t}\right) q\left(N^{(1)}, N^{(2)}\right)=\frac{1}{2} \varepsilon_{1}^{2} \frac{\partial^{2} q}{\partial N^{(1)^{2}}}+\frac{1}{2} \varepsilon_{2}^{2} \frac{\partial^{2} q}{\partial N^{(2)^{2}}} \\
& \quad+\left(\frac{1}{2} k_{1} \varepsilon_{1}^{2} y_{t}^{1}-\Delta\left(N^{(1)}\right)\right) \frac{\partial q}{\partial N^{(1)}}+\left(\frac{1}{2} k_{2} \varepsilon_{2}^{2} y_{1}^{2}-\Delta\left(N^{(2)}\right)\right) \frac{\partial q}{\partial N^{(2)}} \\
& \quad+\left(\frac{1}{2} k_{1}^{2} \varepsilon_{1}^{2}\left(y_{t}^{1}\right)^{2}-k_{1} y_{t}^{1} \Delta\left(N^{(1)}\right)-\frac{1}{2} k_{1}^{2}\left(N_{t}^{(1)}\right)^{2}\right. \\
& \left.\quad+\left(A-2 B N^{(1)}-C N^{(2)}\right)\right) q\left(N^{(1)}, N^{(2)}\right) \\
& \quad+\left(\frac{1}{2} k_{2}^{2} \varepsilon_{2}^{2}\left(y_{1}^{2}\right)^{2}-k_{2} y_{t}^{2} \Delta\left(N^{(2)}\right)-\frac{1}{2} k_{2}^{2}\left(N_{t}^{(2)}\right)^{2}+\left(-D+E N^{(1)}\right)\right) q\left(N^{(1)}, N^{(2)}\right)
\end{aligned}
$$

where

$$
\begin{aligned}
& \Delta\left(N^{(1)}\right)=\left(A-B N^{(1)}-C N^{(2)}\right) N^{(1)} \\
& \Delta\left(N^{(2)}\right)=\left(-D+E N^{(1)}\right) N^{(2)}
\end{aligned}
$$


Let $\left(\tilde{N}_{t}^{(1)}, \tilde{N}_{t}^{(2)}\right)$ be the solution of the system

$$
\tilde{N}_{t}^{(i)}=\tilde{N}_{0}^{(i)}+\int_{0}^{t}\left(\frac{1}{2} k_{i} \varepsilon_{i}^{2} y_{t}^{i}-\Delta\left(\tilde{N}^{i}\right)\right) d s+\varepsilon_{i} d \widetilde{W}_{t}^{i}
$$

Let us now consider the expectation, given $\mathfrak{Y}_{t}$, of

$$
\begin{aligned}
F\left(\tilde{N}_{t}^{(1)}, N_{t}^{(2)}\right) \exp \left\{\int _ { 0 } ^ { t } \sum _ { i = 1 } ^ { 2 } \left(\left(\varepsilon_{i} y^{i} k_{i}\right)^{2}-k_{i} y^{i} \Delta\left(\tilde{N}^{(i)}\right)-\frac{1}{2} k_{t}^{2}\left(\tilde{N}^{(i)}\right)^{2}\right.\right. \\
\left.\left.+\frac{\partial}{\partial N^{(i)}}\left(\Delta\left(\tilde{N}^{(i)}\right)\right)\right) d s\right\}
\end{aligned}
$$

Put

(6.17) $\nu_{t}(F)=E\left(F\left(\tilde{N}_{t}^{(1)}, \tilde{N}_{t}^{(2)}\right)\right.$

$\left.\times \exp \left\{\int_{0}^{t} \sum_{i=1}^{n}\left(\left(\varepsilon_{i} y^{i} k_{i}\right)^{2}-k_{i} y^{i} \Delta\left(\tilde{N}^{(i)}\right)-\frac{1}{2} k_{i}^{2}\left(\tilde{N}^{(i)}\right)^{2}+\frac{\partial}{\partial N^{(i)}}\left(\Delta\left(\tilde{N}^{(i)}\right)\right)\right) d s\right\} \mid \mathfrak{Y}_{t}\right)$,

where

$$
\begin{aligned}
& \frac{\partial}{\partial N^{(1)}}\left(\Delta\left(N^{(1)}\right)\right)=A-2 B N^{(1)}-C N^{(2)} \\
& \frac{\partial}{\partial N^{(2)}}\left(\Delta\left(N^{(2)}\right)\right)=\left(D+E N^{(1)}\right),
\end{aligned}
$$

and the expectation is taken over the backward sample paths. We then have

$$
\nu_{t}(F)=\nu_{0}(F)+\int_{0}^{t} \nu_{s}\left(\mu_{s}^{-1} L^{*}(s) \mu_{s}\right) F\left(N^{(1)}, N^{(2)}\right) d s ;
$$

that is, $\nu_{t}(F)$ solves equation (6.14). Applying Kunita's theorem, the solution of the adjoint Zakai equation is given by

$$
q_{t}(F)=\mu_{t}\left(\nu_{t} F\right)(x)
$$

Evaluating $\mu_{t}\left(\nu_{t} F\right)$, we have

$$
\begin{aligned}
\mu_{t}\left(\nu_{t} F\right)= & \mu_{0}(F)+\int_{0}^{t} L^{*}(s) \mu_{s}\left(\nu_{s}(F)\right) d s+k_{1} \int_{0}^{t} N_{s}^{(1)} \mu_{s}\left(\nu_{s}(F)\right) \circ d W_{s}^{1} \\
& +k_{2} \int_{0}^{t} N_{s}^{(2)} \mu_{s}\left(\nu_{s}(F)\right) \circ d W_{s}^{2} .
\end{aligned}
$$


Hence, the solution of the adjoint Zakai equation for the unnormalised conditional density is given by

$$
\begin{aligned}
q_{t}\left(N_{t}^{(1)}, N_{t}^{(2)}\right)=E & {\left[\exp \sum_{i=1}^{2} k_{i} y^{i} \tilde{N}^{(i)} \cdot F\left(N_{t}^{(1)}, N_{t}^{(2)}\right)\right.} \\
& \times \exp \left\{\int _ { 0 } ^ { t } \left(\sum_{i=1}^{2} \frac{1}{2} k_{i}^{2} \varepsilon_{i}^{2}\left(y_{s}^{i}\right)^{2}-k^{i} y_{s}^{i} \Delta\left(\tilde{N}_{a}^{(i)}\right)-\frac{k_{i}^{2}}{2}\left(\tilde{N}_{s}^{i}\right)^{2}\right.\right. \\
& \left.\left.\left.-k_{i} y_{s}^{i} \frac{\partial}{\partial N^{(i)}}\left(\Delta\left(\tilde{N}^{(i)}\right)\right)\right) d s\right\} \mid \mathfrak{Y}_{t}\right] .
\end{aligned}
$$

Note the appearance of the divergence $\partial /\left(\partial N^{(1)}\right)\left(\left(a-B N^{(1)}-C N^{(2)}\right) N^{(1)}\right)$ and $\partial /\left(\partial N^{(2)}\right)\left(\left(-D+E N^{(1)}\right) N^{(2)}\right)$ in formula $(6.20)$.

This formula is compatible with that obtained for the unnormalised conditional distribution where the expectation is over the forward sample paths and the divergence terms do not appear because there we worked with the Zakai equation itself.

\section{REFERENCES}

[1] P.L. Antonelli (ed.), Mathematical essays on growth and the emergence of form (University of Alberta Press, Edmonton, Canada, 1985).

[2] M.H.A. Davies and S.I. Marcus, 'An introduction to nonlinear filter', in Stochastic Systems: the Mathematics of Filtering and Identification and Applications, (M. Hazewinkel and J.C. Willems, Editors), 1981, pp. 53-75.

[3] R.J. Elliott, Stochastic calculus and applications (Springer-Verlag, Berlin, Heidelberg, New York, 1982).

[4] R.J. Elliott, 'Filtering for a logistic equation', Math. Comput. Modelling 13 (1990), 1-10.

[5] R.E. Kalman and R.S. Bucy, 'New results in linear filtering and prediciton theory', $J$. Basic Eng. 83 (1961), 95-108.

[6] H. Kunita, Stochastic partial differential equations connected with nonlinear filtering, Lecture Notes in Mathematics 972 (Springer-Verlag, Berlin, Heidelberg, New York, 1983).

[7] H.J. Kushner, 'On the differential equations satisfied by conditional probability densities of Markov processes', SIAM J. Control 2 (1964), 106-119.

[8] R.S. Lipster and A.N. Shiryayev, Statistics of random processes I, II (Springer-Verlag, Berlin, Heidelberg, New York, 1977).

[9] B. Oksendal, Stochastic differential equations (Springer-Verlag, Berlin, Heidelberg, New York, 1992).

[10] R.L. Stratonovich, Conditional Markov processes and their applications to the theory of optimal control (Elsevier, New York, 1968).

[11] E. Wong, Stochastic processes in information and dynamical systems (McGraw-Hill, New York, 1971). 
Department of Mathematics and Statistics University of Western Sydney, Macarthur Campbelltown NSW 2560

Australia

e-mail: r.ahlip@uws.edu.au
School of Mathematics

Queensland University of Technology

GPO Box 2434

Brisbane Qld 4001

Australia

e-mail: v.anh@fsc.qut.edu.au 